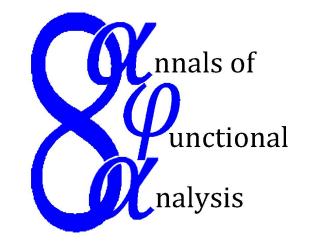

Ann. Funct. Anal. 5 (2014), no. 2, 53-60

$\mathscr{A}$ NNALS OF $\mathscr{F}$ UNCTIONAL $\mathscr{A}$ NALYSIS

ISSN: 2008-8752 (electronic)

URL:www.emis.de/journals/AFA/

\title{
CHARACTERIZATION OF EXPONENTIAL POLYNOMIALS ON COMMUTATIVE HYPERGROUPS
}

\author{
LÁSZLÓ SZÉKELYHIDI \\ This paper is dedicated to Professor Tsuyoshi Ando \\ Communicated by K. Ciesielski
}

\begin{abstract}
Exponential monomials are the basic building bricks of spectral analysis and spectral synthesis on Abelian groups. Recently there have been some attempts to extend the most important spectral analysis and spectral synthesis results from groups to hypergroups. For this purpose it is necessary to introduce a reasonable concept of exponential monomials. In this work we reconsider this problem, and using a ring-theoretical approach we prove characterization theorems for particular function classes, which can be considered as "exponential monomials" on commutative hypergroups.
\end{abstract}

\section{INTRODUCTION AND PRELIMINARIES}

In this paper $\mathbb{C}$ denotes the set of complex numbers. If $K=K(*,, e)$ is a commutative hypergroup, then $\mathcal{C}(K)$ denotes the locally convex topological vector space of all continuous complex valued functions defined on $K$, equipped with the pointwise linear operations and the topology of compact convergence. For each function $f$ in $\mathcal{C}(K)$ we define $\check{f}$ by $\check{f}(x)=f(\check{x})$, whenever $x$ is in $K$.

The dual of $\mathcal{C}(K)$ can be identified with $\mathcal{M}_{c}(K)$, the space of all compactly supported complex measures on $K$. If $K$ is discrete, then this space is also identified with the set of all finitely supported complex valued functions on $K$. The pairing between $\mathcal{C}(K)$ and $\mathcal{M}_{c}(K)$ is given by the formula

$$
\langle\mu, f\rangle=\int f d \mu .
$$

Date: Received: August 7, 2013; Accepted: November 2, 2013.

2010 Mathematics Subject Classification. Primary 20N20; Secondary 43A62, 39B99.

Key words and phrases. Hypergroup, spectral synthesis, exponential polynomial. 
Convolution on $\mathcal{M}_{c}(K)$ is defined by

$$
\mu * \nu(x)=\int \mu(x * \check{y}) d \nu(y)
$$

for any $\mu, \nu$ in $\mathcal{M}_{c}(K)$ and $x$ in $K$. Convolution converts the space $\mathcal{M}_{c}(K)$ into a commutative algebra with unit $\delta_{e}$.

We also define convolution of measures in $\mathcal{M}_{c}(K)$ with arbitrary functions in $\mathcal{C}(K)$ by the same formula

$$
\mu * f(x)=\int f(x * \check{y}) d \mu(y)
$$

for each $\mu$ in $\mathcal{M}_{c}(K), f$ in $\mathcal{C}(K)$ and $x$ in $K$.

Translation with the element $y$ in $K$ is the operator mapping the function $f$ in $\mathcal{C}(K)$ onto its translate $\tau_{y} f$ defined by $\tau_{y} f(x)=f(x * y)$ for any $x$ in $K$. Clearly, $\tau_{y}$ is a convolution operator, namely, it is the convolution with the measure $\delta_{\check{y}}$. A subset of $\mathcal{C}(K)$ is called translation invariant, if it contains all translates of its elements. A closed linear subspace of $\mathcal{C}(K)$ is called a variety on $K$, if it is translation invariant. For each function $f$ the smallest variety containing $f$ is called the variety generated by $f$, or simply the variety of $f$ and is denoted by $\tau(f)$. It is the intersection of all varieties containing $f$.

It is very easy to check that the annihilator of each variety in $\mathcal{C}(K)$ is an ideal in $\mathcal{M}_{c}(K)$, which is proper if and only if the variety is nonzero. The annihilator of the variety $V$ will be denoted by $V^{\perp}$. Analogously, for each ideal $I$ in $\mathcal{M}_{c}(K)$ its annihilator is defined by

$$
I^{\perp}=\{f: f \in \mathcal{C}(K), \mu(f)=0 \text { for each } \mu \in I\} .
$$

It is easy to see that $I^{\perp}$ is a variety in $\mathcal{C}(K)$, which is nonzero if and only if $I$ is proper. It is also obvious that $V \subseteq V^{\perp \perp}$ and $I \subseteq I^{\perp \perp}$ holds for each variety $V$ on $K$ and ideal $I$ in $\mathcal{M}_{c}(K)$. Moreover, using the Hahn-Banach Theorem, it is easy to show that $V=V^{\perp \perp}$ holds for each variety. Although we don't have the corresponding equality for ideals, as it is shown by an example in [3] in the case, when $K$ is a group, however, if $K$ is a discrete hypergroup, then $I=I^{\perp \perp}$ holds for each ideal in $\mathcal{M}_{c}(K)$. This is also shown in [3] in the group-case, and one can see immediately that the proof given there works on hypergroups, too.

The basic problems of spectral analysis and spectral synthesis on groups concern with the description of varieties. For this description the fundamental building bricks are the exponential monomials. Having a reasonable concept of exponential monomials we can formulate these basic problems on hypergroups as follows. Let $K$ be a commutative hypergroup and $V$ a variety on $K$. We say that spectral analysis holds for $V$, if every nonzero subvariety of $V$ contains a nonzero exponential monomial. We say that $V$ is synthesisable, if the exponential monomials in $V$ span a dense subspace. We say that spectral synthesis holds for $V$, if every subvariety of $V$ is synthesisable. Finally, we say that spectral analysis, resp. spectral synthesis holds on $K$, if spectral analysis, resp. spectral synthesis holds 
for each variety on $K$. Recently attempts have been made to introduce a satisfactory concept of exponential monomials on commutative hypergroups. In [5, 6] we proved spectral analysis and spectral synthesis on polynomial hypergroups, calling some particular functions exponential monomials. In [7, 11] exponential monomials have been introduced and studied on Sturm-Liouville hypergroups. In all these cases the common feature of the concept was that the function in question generates a finite dimensional variety. With some additional conditions this concept can be used on arbitrary hypergroups, like in [9], however, it does not seem to be quite satisfactory in the sense that the property generating a finite dimensional variety is too implicit, it does not say useful information on the function itself. In [8] we presented some characterization theorems for exponential monomials, which are more explicit and we proved that the concept given there includes the one on polynomial and on Sturm-Liouville hypergroups used in the references above and it coincides with the one used on Abelian groups.

In this paper we study exponential monomials and exponential polynomials on commutative hypergroups. Here we adopt the way used in [9] of introducing exponential monomials on hypergroups and we prove characterization theorems for these function classes using annihilators. These theorems and the annihilator method applied here are the main results of this work. In particular, we show that the concept of exponential monomial used in [8] coincides with the one on polynomial hypergroups used in [5,6]. We have used similar ideas in [10]. Although the major part of our results holds on arbitrary commutative locally compact hypergroups, in this paper we consider only the discrete case. In the subsequent paragraphs by a hypergroup we always mean a discrete commutative hypergroup. In the discrete group-case $\mathcal{M}_{c}(K)$ is the so-called group algebra of $K$, hence we shall use the notation $\mathbb{C} K$ for it.

For basic knowledge on hypergroups the reader is referred to [1], [7]. Exponential polynomials on special hypergroups have been used in [5, 6, 7]. The concept of exponential polynomials on general commutative hypergroups has been introduced in [8] in a slightly different way.

\section{Generalized exponential monomials}

The basic building bricks of varieties are the exponential monomials. In the group-case exponential monomials are defined as functions generating finite dimensional indecomposable varieties (see e.g. [9]). A variety on a hypergroup is called decomposable, if the algebraic sum of two proper subvarieties is dense in it. Otherwise it is called indecomposable. This property can be characterized by the annihilators due to the following result.

Theorem 2.1. Let $K$ be a hypergroup and $\left(V_{i}\right)_{i \in I}$ a family of varieties on $K$. Then we have

$$
\left(\sum_{i \in I} V_{i}\right)^{\perp}=\bigcap_{i \in I} V_{i}^{\perp}
$$

Proof. Let $\mu$ be in $\left(\sum_{i \in I} V_{i}\right)^{\perp}$, then $\mu$ annihilates each $V_{i}$, hence $\mu$ is in $\bigcap_{i \in I} V_{i}^{\perp}$. Conversely, if $\mu$ belongs to $\bigcap_{i \in I} V_{i}^{\perp}$, then $\mu$ annihilates each $V_{i}$, and also each 
finite sum of these varieties. By continuity, it also annihilates the closure of the union of all finite sums, hence $\mu$ is in $\left(\sum_{i \in I} V_{i}\right)^{\perp}$.

Corollary 2.2. Let $K$ be a hypergroup and $V$ a variety on $K$. Then $V$ is indecomposable if and only if its variety is not the intersection of two ideals different from $V^{\perp}$.

Hence a finite dimensional variety is indecomposable if and only if it is not the sum of two proper subvarieties. The following definition has been used in [8].

Let $K$ be a hypergroup. A function $f$ in $\mathcal{C}(K)$ is called an exponential monomial, if $\tau(f)$ is finite dimensional and indecomposable. The simplest exponential is the one generating a one dimensional variety. A function $m$ in $\mathcal{C}(K)$ is called an exponential, if $\tau(m)$ is one dimensional and $m(e)=1$. The following characterization of exponentials is taken from the results in [8].

Theorem 2.3. Let $K$ be a hypergroup and $f: K \rightarrow \mathbb{C}$ a function. Then the following statements are equivalent.

(1) $f$ is an exponential.

(2) $f$ is a common eigenfunction of all translation operators and $f(e)=1$.

(3) $f$ is a nonzero homomorphism of $K$ into the multiplicative semigroup of complex numbers.

(4) $\tau(f)$ is one dimensional and $f(e)=1$.

(5) $\tau(f)^{\perp}$ is the kernel of a multiplicative functional of the algebra $\mathbb{C} K$ and $f(e)=1$.

(6) The factor algebra $\mathbb{C} K / \tau(f)^{\perp}$ is isomorphic to the algebra of complex numbers and $f(e)=1$.

We note that in contrast to the group-case exponentials on hypergroups may take the value zero.

In [8] we introduced modified differences essentially in the following manner: given a function $f: K \rightarrow \mathbb{C}$ and an element $y$ in $K$, then we let

$$
\Delta_{f ; y}=\delta_{\check{y}}-f(y) \delta_{e} .
$$

For a given function $f: K \rightarrow \mathbb{C}$ the ideal in $\mathbb{C} K$ generated by all modified differences of the form $\Delta_{f ; y}$ with $y$ in $K$ will be denoted by $M_{f}$.

Modified differences are closely related to exponentials as it is shown by the following result.

Theorem 2.4. Let $K$ be a commutative hypergroup and $f: K \rightarrow \mathbb{C}$ a function. Then the following statements are equivalent.

(1) $f$ is an exponential.

(2) The ideal $M_{f}$ is proper and $f(e)=1$.

(3) The ideal $M_{f}$ is maximal and $f(e)=1$.

(4) $M_{f}=\tau(f)^{\perp}$ and $f(e)=1$.

Proof. If $f$ is an exponential, then $f(e)=1$, hence $f \neq 0$. For each $y$ in $K$ we have

$$
\Delta_{f ; y} * f(x)=f(x * y)-f(y) f(x)=0
$$


hence $f$ is in $M_{f}^{\perp}$ and $M_{f}$ is proper.

Suppose that $f(e)=1$ and $M_{f}$ is proper, further let $\varphi \neq 0$ be in $M_{f}^{\perp}$. Then we have for each $x, y$ in $K$

$$
\varphi(x * y)-f(y) \varphi(x)=\Delta_{f ; y} * \varphi(x)=0,
$$

and putting $x=e$ we infer $\varphi=\varphi(e) f$. We infer that $M_{f}^{\perp}$ is one dimensional, hence $M_{f}$ is maximal.

Let $M_{f}$ be maximal and $f(e)=1$. Again, taking $\varphi \neq 0$ in $M_{f}^{\perp}$ we infer that $\varphi=\varphi(e) f$, in particular, $\varphi(e) \neq 0$, hence $f$ is in $M_{f}^{\perp}$, which implies $\tau(f) \subseteq M_{f}^{\perp}$. It follows $M_{f} \subseteq \tau(f)^{\perp}$. As $f \neq 0$, we have that $\tau(f)^{\perp}$ is a proper ideal, and, by the maximality of $M_{f}$ we infer $M_{f}=\tau(f)^{\perp}$.

Finally, suppose that $M_{f}=\tau(f)^{\perp}$ and $f(e)=1$. Then $f$ is in $M_{f}^{\perp}$, hence for each $x, y$ in $K$ we have

$$
0=\Delta_{f ; y} * f(x)=f(x * y)-f(y) f(x),
$$

that is, $f$ is an exponential.

For the products of modified differences we use the notation

$$
\Delta_{m ; y_{1}, y_{2}, \ldots, y_{n+1}}=\prod_{i=1}^{n+1} \Delta_{m ; y_{i}},
$$

for any natural number $n$ and for each $y_{1}, y_{2}, \ldots, y_{n+1}$ in $K$. On the right hand side $\Pi$ is meant as a convolution product.

Modified differences can be used for the characterization of exponential monomials. More generally, in [8] we introduced another basic class of functions. The function $f: K \rightarrow \mathbb{C}$ on $K$ is called a generalized exponential monomial, if there exists an exponential $m$ on $K$ and a natural number $n$ such that for each $y_{1}, y_{2}, \ldots, y_{n+1}$ we have

$$
\Delta_{m ; y_{1}, y_{2}, \ldots, y_{n+1}} * f=0 .
$$

It was shown in [8] that if $f$ is nonzero, then the exponential $m$ in the definition is unique. For the nonzero generalized exponential monomial $f$ we say that it corresponds to the exponential $m$ and we define its degree as the smallest natural number $n$ satisfying (2.1). The zero function is a generalized exponential monomial, too, but its degree is undefined. However, when speaking about generalized exponential monomials of degree at most $n$ for some natural number $n$, we always include the identically zero function, for convenience.

\section{Characterization theorems}

From the definition of generalized exponential monomials we immediately infer the following characterization theorem.

Theorem 3.1. Let $K$ be a hypergroup. The function $f: K \rightarrow \mathbb{C}$ is a generalized exponential monomial if and only if there exists an exponential $m$ and a natural number $n$ such that

$$
M_{m}^{n+1} \subseteq \tau(f)^{\perp} .
$$

Proof. Indeed, the condition given in (2.1) is obviously equivalent to (3.1). 
In [8] we proved that this concept coincides with the usual concept of generalized exponential monomials on commutative groups. Now we show that exponential monomials are generalized exponential monomials.

Theorem 3.2. Exponential monomials are generalized exponential monomials on every hypergroup.

Proof. Let $f: K \rightarrow \mathbb{C}$ be an exponential monomial and suppose that $f \neq 0$. As $\tau(f)$ is finite dimensional, every ascending chain of subvarieties in $\tau(f)$ terminates. This means that every descending chain of ideals containing $\tau(f)^{\perp}$ terminates, in other words, every descending chain of ideals in the ring $\mathbb{C} K / \tau(f)^{\perp}$ terminates, which means that $\mathbb{C} K / \tau(f)^{\perp}$ is an Artin ring. By Theorem 7.15 on p. 426 in [2], Vol. II., $\mathbb{C} K / \tau(f)^{\perp}$ is a direct sum of local Artin rings with nilpotent maximal ideals. It follows

$$
\mathbb{C} K / \tau(f)^{\perp}=\mathbb{C} K / I_{1} \oplus \mathbb{C} K / I_{2} \oplus \cdots \oplus \mathbb{C} K / I_{l}
$$

with some ideals with $\tau(f)^{\perp}=\bigcap_{k=1}^{l} I_{k}$, where $\mathbb{C} K / I_{k}$ is a local Artin ring with nilpotent ideal for $k=1,2, \ldots, l$. As $\tau(f)$ is indecomposable, we have $n=1$ and $\mathbb{C} K / \tau(f)^{\perp}$ is a local Artin ring with nilpotent maximal ideal $\Phi(M)$, where $\Phi: \mathbb{C} K \rightarrow \mathbb{C} K / \tau(f)^{\perp}$ is the natural homomorphism, and $M$ is the unique maximal ideal in $\mathbb{C} K$ including $\tau(f)^{\perp}$. By $M^{\perp} \subseteq \tau(f)$ we conclude that $M^{\perp}$ is a finite dimensional variety, that is, it is a common invariant finite dimensional subspace of all translation operators $\tau_{y}$ for $y$ in $K$. As these operators form a commuting family, we infer that they have a common eigenfunction $m$ in $M^{\perp}$, and, by Theorem 2.3, $m$ is an exponential. Then obviously $M=M_{m}$, and, by the nilpotency of $\Phi(M)$ we obtain $M_{m}^{n+1} \subseteq \tau(f)^{\perp}$. By Theorem 3.1, our theorem is proved.

The following corollary is obvious.

Corollary 3.3. A generalized exponential monomial on a hypergroup is an exponential monomial if and only if it generates a finite dimensional variety.

\section{Generalized exponential polynomials}

Exponential polynomials and generalized exponential polynomials on commutative groups are usually defined as finite sums of exponential monomials, or generalized exponential monomials. In [7] we have used the same concept on hypergroups and here we keep this definition. Our first characterization theorem follows.

Theorem 4.1. Let $K$ be a hypergroup. The function $f: K \rightarrow \mathbb{C}$ is a generalized exponential polynomial if and only if there exist exponentials $m_{1}, m_{2}, \ldots, m_{n}$ and natural numbers $k_{1}, k_{2}, \ldots, k_{n}$ such that

$$
M_{m_{1}}^{k_{1}+1} M_{m_{2}}^{k_{2}+1} \ldots M_{m_{n}}^{k_{n}+1} \subseteq \tau(f)^{\perp} .
$$

Proof. Suppose that $f: K \rightarrow \mathbb{C}$ is a nonzero generalized exponential monomial and

$$
f=\varphi_{1}+\varphi_{2}+\cdots+\varphi_{n}
$$


where the $\varphi_{i}$ 's are nonzero generalized exponential monomials corresponding to different exponentials $m_{i}(i=1,2, \ldots, n)$. Hence there are natural numbers $k_{1}, k_{2}, \ldots, k_{n}$ such that

$$
M_{m_{i}}^{k_{i}+1} \subseteq \tau\left(\varphi_{i}\right)^{\perp}
$$

holds for $i=1,2, \ldots, n$, which implies our statement.

Conversely, suppose that $f$ satisfies the condition of the theorem and the exponentials $m_{1}, m_{2}, \ldots, m_{n}$ are different. Then the ideals $M_{m_{i}}^{k_{i}+1}$ are pairwise coprime, that is, we have

$$
M_{m_{i}}^{k_{i}+1}+M_{m_{j}}^{k_{j}+1}=\mathbb{C} K,
$$

whenever $i \neq j$. Indeed, assuming the contrary there is a maximal ideal $M$ in $\mathbb{C} K$ such that $M_{m_{i}}^{k_{i}+1}+M_{m_{j}}^{k_{j}+1} \subseteq M$, hence $M_{i}^{k_{i}+1} \subseteq M$. As $M$ is a prime ideal, we infer $M_{i} \subseteq M$, and, by maximality, $M_{i}=M$. Similarly, $M_{j}=M$, a contradiction.

As the product of co-prime ideals is equal to their intersection (see e.g. [4], p. 3.), we have

$$
M_{m_{1}}^{k_{1}+1} \cap M_{m_{2}}^{k_{2}+1} \cap \cdots \cap M_{m_{n}}^{k_{n}+1} \subseteq \tau(f)^{\perp} .
$$

By Theorem 2.1 it follows

$$
\tau(f) \subseteq\left(M_{m_{1}}^{k_{1}+1}\right)^{\perp}+\left(M_{m_{2}}^{k_{2}+1}\right)^{\perp}+\cdots+\left(M_{m_{n}}^{k_{n}+1}\right)^{\perp} .
$$

Now our statement follows from the obvious fact that, by Theorem 3.1, the elements of $\left(M_{m_{i}}^{k_{i}+1}\right)^{\perp}$ are generalized exponential monomials.

We have the analogue of Corollary 3.2.

Corollary 4.2. A generalized exponential polynomial on a hypergroup is an exponential polynomial if and only if it generates a finite dimensional variety.

\section{EXPONENTIAL MONOMIALS ON POLYNOMIAL HYPERGROUPS}

In [8] we proved that the concept of exponential monomial, which has been used in [5, 6] is a special case of the concept we use here. More exactly, in [8] we proved the following theorem.

Theorem 5.1. Let $K$ be a polynomial hypergroup generated by the sequence of polynomials $\left(P_{n}\right)_{n \in \mathbb{N}}$. Then for each complex number $\lambda$ and natural number $k$ the function $n \mapsto P_{n}^{(k)}(\lambda)$ is an exponential monomial of degree at most $k$ corresponding to the exponential $n \mapsto P_{n}(\lambda)$.

Now we prove a more general characterization theorem.

Theorem 5.2. Let $K=\left(Q_{x}, *\right)$ be a polynomial hypergroup in d dimension, and $\lambda$ an arbitrary element in $\mathbb{C}^{d}$. Then the function $f: K \rightarrow \mathbb{C}$ is an exponential monomial corresponding to the exponential $x \mapsto Q_{x}(\lambda)$ if and only if it is a linear combination of the functions of the form $x \mapsto P(\partial) Q_{x}(\lambda)$, where $P: \mathbb{C}^{d} \rightarrow \mathbb{C}$ is a polynomial.

Proof. As $(x, \lambda) \mapsto Q_{x}(\lambda$ is an exponential family for $K$ (see [7], the sufficiency follows from Lemma 3 in [8]. To prove the converse suppose that $f: K \rightarrow \mathbb{C}$ is an exponential monomial corresponding to the exponential $x \mapsto Q_{x}(\lambda)$. Then $\tau(f)$ is finite dimensional. In [6] we have proved (Theorem 5) that in each variety 
on $K$ the functions of the form $x \mapsto P(\partial) Q_{x}(\lambda)$ span a dense subspace, where $P: \mathbb{C}^{d} \rightarrow \mathbb{C}$ is a polynomial and $\lambda$ is in $\mathbb{C}^{d}$. It follows that each finite dimensional variety is the linear span of functions of this form. We only have to show that in $\tau(f)$ all functions of this form have the same $\lambda$, that means, all these functions correspond to the same exponential $x \mapsto Q_{x}(\lambda)$. But this follows from the fact that $\tau(f)^{\perp}$ is included in exactly one maximal ideal, as we have shown in the proof of Theorem 3.2.

Acknowledgement The research was supported by the Hungarian National Foundation for Scientific Research (OTKA), Grant No. NK-81402.

\section{REFERENCES}

1. W.R. Bloom and H. Heyer, Harmonic analysis of probability measures on hypergroups, de Gruyter Studies in Mathematics, vol. 20, Walter de Gruyter \& Co., Berlin, 1995.

2. N. Jacobson, Basic Algebra, II., W. H. Freeman and Company, New York, 1989.

3. M. Laczkovich and L. Székelyhidi, Spectral synthesis on discrete Abelian groups, Math. Proc. Camb. Phil. Soc. 143(1)(2007), 103-120.

4. H. Matsumura, Commutative ring theory, Cambridge Studies in Advanced Mathematics, vol. 8, Cambridge University Press, Cambridge, 1989.

5. L. Székelyhidi, Spectral analysis and spectral synthesis on polynomial hypergroups, Monatsh. Math. 141 (2004), no. 1, 33-43.

6. L. Székelyhidi, Spectral synthesis on multivariate polynomial hypergroups, Monatsh. Math. 153 (2008), no. 2, 145-152.

7. L. Székelyhidi, Functional Equations on Hypergroups, World Scientific Publishing Co. Pte. Ltd., Singapore, New Jersey, London, Hong Kong, 2012.

8. L. Székelyhidi, Exponential polynomials on commutative hypergroups, Arch. der Math. (to appear).

9. L. Székelyhidi, Vector modules and spectral synthesis, Banach Center Publications (to appear).

10. L. Székelyhidi, A characterization of exponential polynomials, Publ. Math. Debrecen (to appear).

11. L. Vajday, Exponential monomials on Sturm-Liouville hypergroups, Banach J. Math. Anal. 4 (2010), no. 2, 139-146.

Institute of Mathematics, University of Debrecen, H-4010 Debrecen, Pf 12., HUNGARY

Department of Mathematics, University of Botswana, Botswana.

E-mail address: 1szekelyhidi@gmail.com 